\title{
A Generalization OF PEANO'S EXISTENCE THEOREM AND FLOW INVARIANCE
}

\author{
MICHAEL G. CRANDALL ${ }^{1}$
}

\begin{abstract}
Let $F \subseteq R^{n}$ be closed and $A: F \rightarrow R^{n}$ be continuous. Assuming that for $y \in F$ the distance from $y+h A y$ to $F$ is $o(h)$ as $h \downarrow 0$, it is shown that for each $x \in F$ the Cauchy problem $u^{\prime}=A u$, $u(0)=x$, has a solution $u:\left[0, T_{x}\right] \rightarrow F$ on some interval $\left[0, T_{x}\right], T_{x}>0$.
\end{abstract}

Throughout this note $F$ is a subset of $R^{m}, A: D(A) \rightarrow R^{m}$ is a continuous function with domain $D(A)$, and $F \subseteq D(A) \subseteq R^{m}$. $B_{r}(x)$ is the closed ball of radius $r$ and center $x$ in $R^{m}$. We will always assume that $F$ is locally closed, i.e. for each $x \in F$ there is an $r>0$ such that $F \cap B_{r}(x)$ is closed in $R^{m}$. The euclidean norm of $y \in R^{m}$ is denoted by $|y|$ and $|y, F|$ stands for the distance from $y$ to $F$. Our main result concerns the Cauchy problem

$$
u^{\prime}=A u, \quad u(0)=x .
$$

By a solution of (1) on an interval $[0, a], a>0$, we mean a continuously differentiable function $u:[0, a] \rightarrow D(A)$ such that $u(0)=x$ and $u^{\prime}(t)=$ $A u(t)$ for $0 \leqq t \leqq a$.

THEOREM 1. Let

$$
\lim _{h \downarrow 0} h^{-1}|z+h A z, F|=0 \text { for } z \in F .
$$

Then for each $x \in F$ there is a positive number $T$ and a solution $u$ of (1) on $[0, T]$ such that $u(t) \in F$ for $0 \leqq t \leqq T$.

This theorem is related to results of Bony [1] and Brezis [2]. We say that $F$ is forward invariant for (1) if whenever $u$ is a solution of (1) on $[0, a], a>0$, and $x \in F$, then $u(t) \in F$ for $0 \leqq t \leqq a$. If $D(A)$ contains a neighborhood of $F$ and $F$ is forward invariant for (1), Theorem 1 is an obvious consequence of the Peano existence theorem. Brezis established that $F$ is forward invariant for (1) if $F$ is closed, $\left(\mathrm{A}_{1}\right)$ holds and $A$ is Lipschitz continuous. Bony replaced $\left(\mathrm{A}_{1}\right)$ with a subtler condition. Let $x \in F$ and $y \in R^{m}$. If the interior of $B_{|y-x|}(y)$ does not meet $F$ we say that $y-x$ is a normal to $F$ at $x$ in the sense of Bony. Let $v(x)$ be the set of such

Received by the editors April 25, 1972.

AMS 1970 subject classifications. Primary 34G05.

${ }^{1}$ Sponsored by the U.S. Army under Contract No. DA-31-124-ARO-D-462. 
normals for $x \in F$. Bony showed that if $F$ is closed, $A$ is Lipschitz continuous and

$$
(z, A x) \leqq 0 \text { for } x \in F \text { and } z \in v(x),
$$

then $F$ is forward invariant for (1). Here ( , ) denotes the euclidean inner-product. Theorem 1 goes beyond such considerations in that it asserts the existence of solutions $u$ with values in $F$ in a generality which allows $F$ to fail to be forward invariant for (1). Moreover, if $F$ is closed and solutions of (1) for $x \in F$ are locally (forward) unique, then it follows at once from Theorem 1 that $F$ is forward invariant for (1). This result generalizes the results of Brezis and Bony. Moreover, it settles a problem mentioned in Redheffer [5]. The results of Brezis and Bony are extended and simplified in [5].

Our proof is a simple adaptation of the method of polygonal approximation. The success of the argument rests on Lemma 1 which shows that if $\left(\mathrm{A}_{1}\right)$ holds, then it holds uniformly on compact subsets of $F$. It is obvious that $\left(A_{1}\right) \Rightarrow\left(A_{2}\right)$. Moreover, it is clear that if the conclusion of Theorem 1 holds, then $\left(A_{1}\right)$ holds (see, e.g., [2]). Lemma 1 actually asserts that if $\left(A_{2}\right)$ holds, then $\left(\mathrm{A}_{1}\right)$ holds uniformly on compact subsets of $F$, which does not seem obvious. As regards Theorem 1, see $\S 5$ of [4].

In [4] R. H. Martin carries out related investigations in a more complex infinite dimensional setting under assumptions which guarantee uniqueness of solutions of (1).

Proof of Theorem 1. Let $x \in F$ and $F_{r}=B_{r}(x) \cap F$. Choose $r>0$ so that $F_{2 r}$ is closed and set

$$
M=\max \left(\max \left\{|A x|: x \in F_{2 r}\right\}, 1\right), \quad T=r / 3 M .
$$

For each integer $n>0$, set $x_{n, 0}=x$ and inductively choose $x_{n, i} \in F$, $1 \leqq i \leqq n$, satisfying

$$
2\left|x_{n, i}+(T / n) A x_{n, i}, F\right| \geqq\left|x_{n, i+1}-\left(x_{n, i}+(T / n) A x_{n, i}\right)\right| .
$$

The existence of $\left\{x_{n, i}\right\}_{i=0}^{n}$ is obvious once we show that if $x_{n, i}$ is defined for $0 \leqq i \leqq k \leqq n$ and $x_{n, i} \in F_{r}$ for $0 \leqq i<k$, then $x_{n, k} \in F_{r}$. If $z \in F_{2 r}$, then

$$
|z+(T / n) A z, F| \leqq|(z+(T / n) A z)-z| \leqq(T / n) M .
$$

It then follows from (3) that

$$
\left|x_{n, i+1}-x_{n, i}\right| \leqq(3 T / n) M
$$

for $0 \leqq i \leqq k-1$. Hence

$$
\left|x_{n, k}-x\right| \leqq \sum_{i=0}^{k-1}\left|x_{n, i+1}-x_{n, i}\right| \leqq(k 3 T / n) M \leqq 3 M T \leqq r .
$$


To continue, define $u_{n}:[0, T] \rightarrow R^{n}$ by

$$
u_{n}(t)=x_{n, i}+(t-i T / n)(n / T)\left(x_{n, i+1}-x_{n, i}\right)
$$

for $i T / n \leqq t \leqq(i+1) T / n, 0 \leqq i \leqq n-1$. Each $u_{n}$ is a continuous piecewise linear mapping, $u_{n}(0)=x$, and by $(4)$,

$$
\left|u_{n}^{\prime}(t)\right| \leqq 3 M
$$

for $0 \leqq t \leqq T$ and $t \notin\{i T / n: i=0,1, \cdots, n\}$. It follows at once from the Arzela-Ascoli theorem that $\left\{u_{n}\right\}_{n=1}^{\infty}$ has a subsequence $\left\{u_{n(k)}\right\}_{k=1}^{\infty}$ which converges uniformly on $[0, T]$ to a limit $u(t)$. Clearly $\left|u_{n}(t), F\right| \leqq 3 M T / n$ for $t \in[0, T]$, so $|u(t), F| \leqq 0$. Since $\left|u_{n}(t)-x\right| \leqq 3 M T \leqq r$ for $0 \leqq t \leqq T$, $u(t) \in B_{r}(x)$. Thus $|u(t), F|=\left|u(t), F_{2 r}\right|$. Since $F_{2 r}$ is closed $u(t) \in F$. Let $D_{r}$ denote the right derivative. Since $D_{r} u_{n}(t)=(n / T)\left(x_{n, i+1}-x_{n, i}\right)$ for $i T / n \leqq t<(i+1) T / n$ it will follow easily that $\lim _{k \rightarrow \infty}\left|D_{r} u_{n(k)}(t)-A u(t)\right|=0$ holds uniformly in $t, 0 \leqq t<T$, if we show

$$
\lim _{n \rightarrow \infty} \max _{0 \leqq i \leqq n-1}\left|(n / T)\left(x_{n, i+1}-x_{n, i}\right)-A x_{n, i}\right|=0 .
$$

Thus the proof that $u(t)$ is a solution of (1) is completed by verifying (7), which is established with the aid of:

LemMA 1. Let $A$ satisfy $\left(\mathrm{A}_{2}\right)$. Then $\left(\mathrm{A}_{1}\right)$ holds uniformly on every compact subset of $F$.

Proof. Let $C \subseteq F$ be compact. Since $F$ is locally closed, $C$ has a compact neighborhood $K$ in $R^{n}$ such that $K \cap F=C_{1}$ is compact. There is an $h_{0}>0$ such that $|x+h A x, F|=\left|x+h A x, C_{1}\right|$ for $x \in C$ and $0 \leqq h \leqq h_{0}$. We assume $0 \leqq s, \tau \leqq h_{0}$ everywhere below. Let $x \in C$ and $y_{\tau}=x+\tau A x$. Choose $x_{\tau} \in C_{1}$ such that $\left|y_{\tau}, F\right|=\left|y_{\tau}-x_{\tau}\right|$. Set

$$
\gamma(r)=\sup \left\{|A z-A y|: z, y \in C_{1} \text { and }|z-y| \leqq r\right\}
$$

and

$$
f(\tau)=\left|y_{\tau}, F\right|^{2} .
$$

Let $0 \leqq s<\tau \leqq h_{0}$. Then, using (8) and (9),

$$
\begin{aligned}
f(\tau)-f(s)= & \left|y_{\tau}-x_{\tau}\right|^{2}-\left|y_{s}-x_{s}\right|^{2} \leqq\left|y_{\tau}-x_{s}\right|^{2}-\left|y_{s}-x_{s}\right|^{2} \\
= & \left|y_{\tau}-y_{s}\right|^{2}+2\left(y_{\tau}-y_{s}, y_{s}-x_{s}\right) \\
= & (\tau-s)^{2}|A x|^{2}+2(\tau-s)\left(A x_{s}, y_{s}-x_{s}\right) \\
& +2(\tau-s)\left(A x-A x_{s}, y_{s}-x_{s}\right) \\
\leqq & (\tau-s)^{2}|A x|^{2}+2(\tau-s)\left(A x_{s}, y_{s}-x_{s}\right) \\
& +2(\tau-s) \gamma\left(\left|x-x_{s}\right|\right) \sqrt{ }(f(s)) .
\end{aligned}
$$


Next observe that $y_{s}-x_{s} \in v\left(x_{s}\right)$ and

$$
\left|x-x_{s}\right| \leqq\left|x-y_{s}\right|+\left|y_{s}-x_{s}\right| \leqq 2\left|x-y_{s}\right|=2 s|A x| .
$$

Hence (by $\left.\left(\mathrm{A}_{2}\right)\right)\left(A x_{s}, y_{s}-x_{s}\right) \leqq 0$ and $\gamma\left(\left|x-x_{s}\right|\right) \leqq \gamma(2 s|A x|)$. Using these estimates in (10), dividing by $(\tau-s)$ and letting $s \uparrow \tau$ yields

$$
\underset{s i \tau}{\lim \sup } \frac{f(\tau)-f(s)}{\tau-s} \leqq 2 \gamma(2 \tau|A x|) \sqrt{ }(f(\tau))
$$

It follows at once that

$$
\sqrt{ }(f(h))=|x+h A x, F| \leqq \int_{0}^{h} \gamma(L s) d s
$$

where $L=2 \max \left\{|A x|: x \in C_{1}\right\}$. Since $A$ is uniformly continuous on $C_{1}$, $\lim _{r \downarrow 0} \gamma(r)=0$. Thus the right-hand side of $(11)$ is $o(h)$ as $h \downarrow 0$, and the proof is complete.

We finish the proof of Theorem 1. Set $\eta(\tau)=\sup \left\{|y+\tau A y, F|: y \in F_{\tau}\right\}$. Since $\left(\mathrm{A}_{1}\right) \Rightarrow\left(\mathrm{A}_{2}\right)$, Lemma 1 implies that $\lim _{\tau \downarrow 0} \eta(\tau) / \tau=0$. Since $x_{n, i} \in F_{\tau}$ for $0 \leqq i \leqq n,(3)$ implies

$$
2 \eta(T / n) n / T \geqq\left|(n / T)\left(x_{n, i+1}-x_{n, i}\right)-A x_{n, i}\right|
$$

and (7) follows at once. The proof is complete.

REMARK 1. Let $E \subseteq R^{m}$ and $B:[a, b) \times E \rightarrow R^{m}$ be continuous and satisfy

$$
\lim _{h \downarrow 0} h^{-1}|y+h B(t, y), E|=0 \quad \text { for } t \in[a, b), y \in E .
$$

Let $F=[a, b) \times E$ and $A: F \rightarrow R^{m+1}$ be defined by $A(t, y)=(1, B(t, y))$ for $(t, y) \in F$. If $t \in[a, b)$,

$$
|(t, y)+h A(t, y), F|=|y+h B(t, y), E|
$$

provided $h \geqq 0$ is sufficiently small. Thus $A$ satisfies the assumptions of Theorem 1 . Moreover, if $E$ is locally closed (or closed), $F$ is locally closed. In the usual way, we conclude:

THEOREM 2. Let $E$ be locally closed and $B:[a, b) \times E \rightarrow R^{m}$ be continuous and satisfy $\left(\mathrm{B}_{1}\right)$. If $(\tau, z) \in[a, b) \times E$, then there is a $T, 0<T<b-\tau$, and $a$ continuously differentiable function $u:[\tau, \tau+T] \rightarrow E$ such that $u(\tau)=z$ and $u^{\prime}(t)=B(t, u(t))$ for $t \in[\tau, \tau+T]$.

REMARK 2. The proof of Lemma 1 shows that $\left(\mathrm{A}_{2}\right) \Rightarrow\left(\mathrm{A}_{1}\right)$ if $F$ is a locally weakly closed subset of a Hilbert space $H$ and $A: F \rightarrow H$ is continuous. 
REMARK 3. The author proved Lemma 1 in 1971 in response to a query of $R$. Redheffer concerning the implication $\left(A_{2}\right) \Rightarrow\left(A_{1}\right)$. The proofs of Lemma 1 of the current paper and Theorem 1 of [5], which are related, were obtained independently.

REMARK 4. After the preparation of this note, P. Hartman kindly provided the author with a preprint of the paper [3] which contains, among other interesting results, a version of Theorem 1. Hartman's proof of Theorem 1 is shorter than that given here, but it does not include the fact that Theorem 1 is valid under the assumption $\left(A_{2}\right)$ in place of $\left(A_{1}\right)$. Certain convexity assumptions employed in [3] can be eliminated by using the normals $v(x)$ employed here and Lemma 1. For this purpose, it is worth noting that $v(x)$ is closed, convex and $r v(x) \subset v(x)$ for $0 \leqq r \leqq 1$.

\section{REFERENCES}

1. J.-M. Bony, Principe du maximum, inéqualité de Harnack et unicité du problèmes de Cauchy pour les opérateurs elliptiques dégénérés, Ann. Inst. Fourier Grenoble 19 (1969), 277-304.

2. H. Brezis, On a characterization of flow-invariant sets, Comm. Pure Appl. Math. 23 (1970), 261-263. MR 41 \#2161.

3. P. Hartman, On invariant sets and on a theorem of Ważewski, Proc. Amer. Math. Soc. 32 (1972), 511-520.

4. R. H. Martin, Differential equations on closed subsets of a Banach space, Trans. Amer. Math. Soc. (to appear).

5. R. M. Redheffer, The theorems of Bony and Brezis on flow-invariant sets, Amer. Math. Monthly (to appear).

Mathematics Research Center, University of Wisconsin, Madison, Wisconsin 53706

Department of Mathematics, University of California, los Angeles, CaliFORNIA 90024 (Current address) 